\title{
Complete resolution of chylopericardium after chemotherapy for chronic lymphocytic leukemia
}

\author{
A.L. Morris $\mathrm{MD}^{* \dagger}$ T. Colbourne $\mathrm{MD}_{1}^{\dagger}$ I. Kirkpatrick $\mathrm{MD}^{,+\ddagger}$ and V. Banerji $\mathrm{MD}^{+\S}$
}

\begin{abstract}
Nontraumatic chylous pleural effusions (chylothorax) and pericardial effusions (chylopericardium) are rare. They can, however, accompany intrathoracic malignancies and, most commonly, lymphomas. An association of chronic lymphocytic leukemia (CLL) with chylopericardium has rarely been reported.

A 68-year-old woman with CLL, previously treated with single-agent fludarabine in the community, developed pleuritic chest pain and a new pericardial effusion. Computed tomography (ст) imaging of her chest revealed a large pericardial effusion with progressive lymphadenopathy. Pericardiocentesis identified a chylous effusion, and complete evacuation was achieved by catheter drainage. The CLL was not treated.

An asymptomatic pericardial effusion subsequently recurred. Pericardiocentesis was not repeated. Lymph node biopsy and flow cytometry revealed no evidence of large-cell lymphoma transformation. The patient was treated with 6 cycles of chlorambucil and obinutuzumab. Imaging of her chest by ст between cycles 2 and 3 revealed a marked resolution of the intrathoracic lymphadenopathy, with complete disappearance of the pericardial effusion. Repeat imaging at 5 months and again at 3 years after completion of chemotherapy demonstrated no recurrence of either the lymphadenopathy or the pericardial effusion.

The mechanism of production and the treatment of chylous effusions are poorly defined. In this case, resolution of the pericardial effusion with effective chemotherapy is postulated to have alleviated obstruction of anterograde lymphatic flow facilitating drainage into the systemic venous system and allowing for spontaneous complete resolution of the pericardial effusion without surgical intervention.
\end{abstract}

Key Words Chylopericardium, pericardial effusion, chronic lymphocytic leukemia, chylous effusion, pericardiocentesis

www.current-oncology.com

\section{INTRODUCTION}

Nontraumatic chylous pleural effusions (chylothorax) and pericardial effusions (chylopericardium) are rare. They can, however, accompany intrathoracic malignancies and, most commonly, lymphomas. An association of chronic lymphocytic leukemia (CLL) with chylopericardium has rarely been reported.

\section{CASE DESCRIPTION}

A 68-year-old woman with known chronic lymphocytic leukemia (CLL) was evaluated for pleuritic chest pain and was found to have a pericardial effusion with no evidence of pulmonary embolism. She reported a nonproductive cough and night sweats. Fevers, hemoptysis, pneumonias, dyspnea, palpitations, abdominal discomfort, edema, and weight loss were denied. There was no history of pericarditis or of collagen vascular disease.

The patient looked well. Blood pressure was 146/70 mmHg. Her pulse was $78 \mathrm{bpm}$ and regular. Jugular venous pressure was normal, and no edema was evident. Her lungs were clear. There were no friction rubs. A 1/6 aortic insufficiency murmur at the right upper sternal border was noted. Liver and spleen were normal.

Computed tomography (Ст) imaging of the chest [Figure 1(B)] revealed a large pericardial effusion that had not been present 3 years earlier. Progression of prior maxillary, mediastinal, and hilar adenopathy, with mild focal consolidation at the left lung base, were evident [Figure 1(A)]. An echocardiogram revealed normal right and left ventricles. There was mild aortic valve sclerosis, with mild aortic 
valve insufficiency. No hemodynamic compromise from a moderate-size echolucent pericardial effusion was evident. An electrocardiogram was normal.

Serum creatinine and troponin T were normal. A white blood cell count was $14.8 \times 10^{-9} / \mathrm{L}$, with $72 \%$ lymphocytes. Hemoglobin was $122 \mathrm{~g} / \mathrm{L}$, and platelets were $135 \times 10^{-9} / \mathrm{L}$.

Pericardiocentesis and catheter drainage revealed a chylous effusion with a triglyceride level of $12.9 \mathrm{mmol} / \mathrm{L}$. The effusion was completely evacuated.

Analysis of the pericardial fluid revealed a cell count of $18.4 \times 10^{-9} / \mathrm{L}$, with $98 \%$ lymphocytes and $2 \%$ monocytes. Flow cytometry revealed $32 \%$ cLL cells, monoclonal B cell immunophenotype CD19+, CD20+, CD5+, CD10-, CD38+, CD43+, CD71-, CD23+.

An asymptomatic pericardial effusion recurred. Pericardiocentesis was not repeated. Imaging by positronemission tomography demonstrated diffuse lymphadenopathy and intense hypermetabolic activity of the lymph nodes in the anterior mediastinum and left axilla (7.2-11.8 standardized uptake value). Findings from a biopsy at the site of the highest standardized uptake
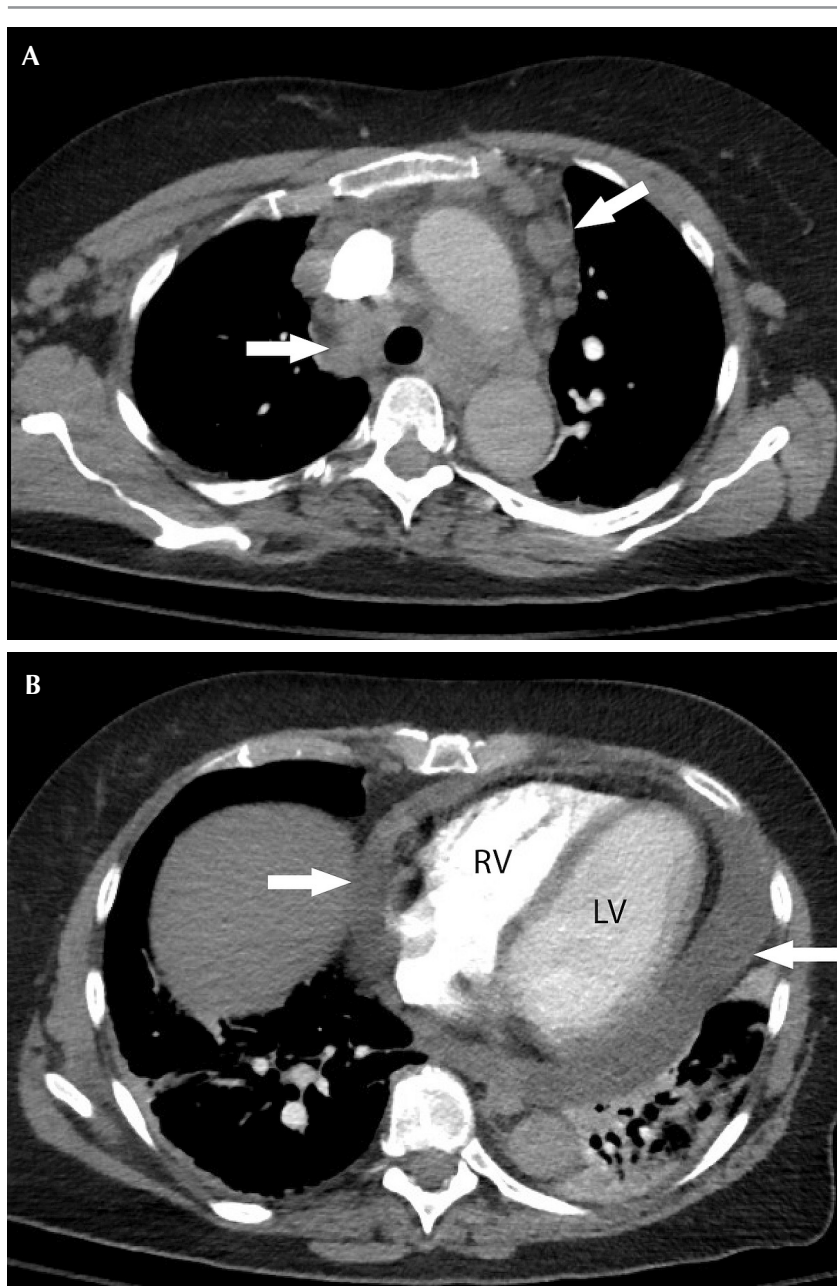

FIGURE 1 (A) Transverse image from an intravenous contrastenhanced computed tomography image of the chest just below the aortic arch shows extensive mediastinal (arrows) and axillary lymphadenopathy. (B) More inferior image shows a moderately large pericardial effusion (arrows). $L V=$ left ventricle; RV = right ventricle. value were consistent with CLL and not with diffuse large B cell lymphoma.

Because the patient had never been treated with a monoclonal antibody, she received 6 cycles of chlorambucil and obinutuzumab. Further ст imaging obtained between chemotherapy cycles 2 and 3 (Figure 2) revealed complete resolution of the pericardial effusion and marked improvement of the lymphadenopathy.

Three years after treatment, the patient remained in remission with no imaging evidence of lymphadenopathy and no pleural or pericardial effusions.

\section{DISCUSSION}

Embryologically, the lymphatic system develops in association with the venous system ${ }^{1}$ and therefore follows the diffuse, complex course of the veins. Chyle, from the Greek chylos ("juice"), enters the lacteals and drains superiorly
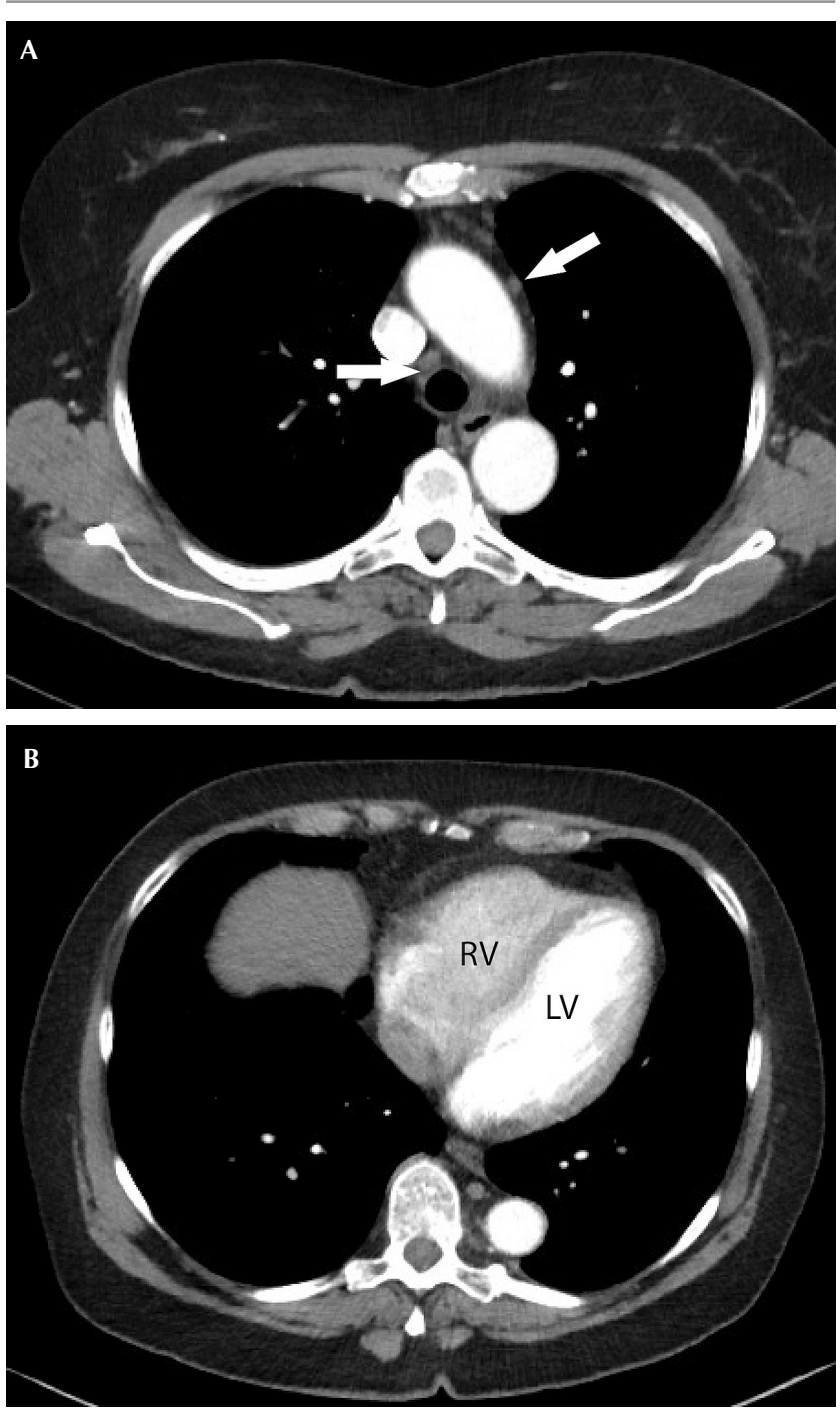

FIGURE 2 (A) Follow-up intravenous contrast-enhanced computed tomography imaging after chemotherapy shows marked improvement, with only a few tiny nodes remaining (arrows). (B) The pericardial effusion has completely resolved. LV = left ventricle; RV = right ventricle. 
into the cisterna chyli to reach the thoracic duct and, subsequently, the systemic veins in the neck. Obstruction of flow in the thoracic duct or trauma to the thoracic duct or its branches can lead to either regurgitation of the flow of chyle into proximal thoracic lymphatics or to leakage from ruptured conduits, or both.

All areas of the body have an extensive-and complex-lymphatic network, with multiple inter-lymphatic and lymphatic-venous connections. The lymphatic system has been well delineated by cт lymphangiography ${ }^{2-4}$, and that technique has been used to attempt to identify points of leakage or obstruction of lymphatic flow.

Given the rarity of both chylothorax and chylopericardium $^{5-10}$, the necessary and sufficient anatomic and physiologic circumstances for the development of such chylous effusions have not been defined. Thoracic duct ligation studies in dogs ${ }^{11}$ demonstrated recruitment of lymphaticovenous connections with the potential for flow of chyle into systemic veins despite the thoracic duct obstruction. Retrograde lymphatic flow was postulated to occur secondary to dilatation of the lymphatics, resulting in incompetence of the lymphatic valves.

Chylothorax, a chylous pleural effusion, is a rare entity most often caused by malignant tumours or traumatic injury to the thoracic duct. Valentine and Raffin ${ }^{12}$ reported that $46 \%$ of patients with chylothorax had malignant tumours and $28 \%$ had experienced traumatic injury; in $14 \%$ of the patients, the causes were idiopathic. Malignant lymphoma constituted $70 \%$ of the neoplastic group.

Chylopericardium, a chylous pericardial effusion, is rarer than chylothorax ${ }^{13}$. The entity was first reported by Hasebrock $^{14}$ in 1888 and was reviewed in 1935 by Yater ${ }^{13}$. The latter author identified only 3 cases of chylopericardium among 100 cases of nontraumatic chylothorax reported in the literature. The review did not mention CLL.

The appearance of chylopericardium in association with CLL has rarely been reported. The Mayo Clinic ${ }^{6}$ reviewed the 33 published cases of chylopericardium in adults during a 10-year interval (1996-2006). The most common cause, in $56 \%$ of cases, was idiopathic (a group that included abnormalities of the lymphatic system and mediastinal lymphangiectasis $)^{8,15}$. The next most common category, representing $15 \%$ of cases, was cardiac surgery associated with trauma to the thoracic duct or its branches. Malignant disease of the lymphatic system or other mediastinal neoplasms accounted for only $6 \%$ of cases. Neither lymphoma nor CLL was noted in the Mayo Clinic's 10-year literature review.

Our review of the literature concerning chylothorax and chylopericardium associated with CLL between 2005 and 2018 (that is, since the report from Dib et al. ${ }^{6}$ ) revealed 15 reports of chylothorax and none of chylopericardium. Both phenomena continue to remain rare. The relatively more frequent association of chylothorax with various lymphomas compared with cLL might be hypothesized to be a result of the greater severity and extent of intrathoracic lymphomatous involvement with lymphomas. Notwithstanding, the necessary and specific criteria for the development of chylous effusions in either of those disorders remain undefined and must explain why both complications remain so rare.
We assume that retrograde lymphatic flow proximal to the site of obstruction, in association with secondary lymphatic valve incompetence, permitted the flow of chyle into the pericardial lymphatics and weeping of chyle into the pericardial space. Thoracic duct obstruction, however produced, does not invariably lead to either chylothorax or chylopericardium ${ }^{11}$. Any postulated mechanism for the development of such effusions must therefore account for the rarity of the phenomena and must explain why such effusions do not occur more often in patients with intrathoracic neoplasms of multiple types. It is likely that a single site of obstruction is not sufficient to produce reflux of chyle into either the pleural or the pericardial space and that other-poorly understood-mechanisms must be postulated to be simultaneously operative.

In our patient, the extent of mediastinal lymph node enlargement might have impaired cephalad drainage of the pericardial lymphatics while simultaneously precluding development of collateral drainage pathways. Whatever the mechanism for the production of chylopericardium in our patient with CLL, the marked resolution of the intrathoracic lymphadenopathy with chemotherapy was associated with complete resolution of the pericardial effusion. Medical and surgical treatment options for persistent chylothorax and chylopericardium never had to be considered ${ }^{3,6}$.

\section{SUMMARY}

The treatment with chlorambucil and obinutuzumab of CLL complicated by chylopericardium has never been reported. An extensive evaluation of our patient failed to demonstrate diffuse large B cell lymphoma, which was suspected to have been the statistically more likely cause of chylopericardium (with the latter condition being more often related to aggressive lymphomas than to CLL, as already noted). The patient's positron-emission tomography-avid disease led to a directed biopsy that confirmed CLL. Because the patient has been in remission for 3 years with no recurrence of the effusion and no evidence of recurrent nodal disease, her clinical course has also been inconsistent with an aggressive lymphoma and remains consistent with CLL. Given the rarity of that confluence (that is, chylopericardium and CLL), diagnostic studies to eliminate the possibility of lymphoma are essential to ensure that the appropriate treatment is provided.

\section{ACKNOWLEDGMENTS}

We thank Ms. Maureen Babb вA MLIs, University of Manitoba, and Ms. Angela Ramjiawan MSc, Office of Clinical Research, St. Boniface Hospital, for expert help in the preparation of the manuscript.

\section{CONFLICT OF INTEREST DISCLOSURES}

We have read and understood Current Oncology's policy on disclosing conflicts of interest, and we declare that we have none.

\section{AUTHOR AFFILIATIONS}

*St. Boniface Hospital; ${ }^{\dagger}$ Department of Internal Medicine and ${ }^{\ddagger}$ Department of Radiology, Max Rady College of Medicine, University of Manitoba; and ${ }^{\S}$ CancerCare Manitoba, Winnipeg, MB.

\section{REFERENCES}

1. Cardiovascular System Development [Web page]. In: Hill MA. Embryology [Web wiki-type resource]. Sydney, Australia: 
University of New South Wales; 2019. [Available at: https:// embryology.med.unsw.edu.au/embryology/index.php/ Cardiovascular_System_Development; cited 5 November 2018]

2. Sehgel R, Schriver E, Tsapenko A, Turner J. Superior vena cava syndrome, chylothorax, chylopericardium: a rarely reported finding in non-small cell lung cancer [abstract]. Chest 2017;152(suppl):A655.

3. Kawasaki R, Sugimoto K, Fujii M, et al. Therapeutic effectiveness of diagnostic lymphangiography for refractory postoperative chylothorax and chylous ascites: correlation with radiologic findings and preceding medical treatment. AJR Am J Roentgenol 2013;201:659-66.

4. Guermazi A, Brice P, Hennequin C, Sarfati E. Lymphography: an old technique retains its usefulness. Radiographics 2003;23:1541-58.

5. Andrade Santiago J, Robles L, Casimiro C, et al. Chylopericardium of neoplastic aetiology. Ann Oncol 1998;9:1339-42.

6. Dib C, Tajik AJ, Park S, Kheir ME, Khandieria B, Mookadam F. Chylopericardium in adults: a literature review over the past decade (1996-2006). J Thorac Cardiovasc Surg 2008;136:650-6.
7. Kohmoto O, Kawabe K, Ono H, et al. Chylothorax associated with chronic lymphocytic leukemia. Intern Med 2016;55:3641-4.

8. Yu X, Jia N, Ye S, Zhou M, Liu D. Primary chylopericardium: a case report and literature review. Exp Ther Med 2018;15:419-25.

9. Zimhony O, Davidovitch Y, Shtalrid M. Chronic lymphocytic leukemia complicated by chylothorax. J Int Med 1994;235:375-7.

10. Hsu CY, Huang LY, Chan JS, Yang CC, Hsiao PJ. Chylopericardium. QJM 2018;111:203-4.

11. Takashima T, Benninghoff DL. Experimental thoracic duct obstruction in dogs. Jpn Circ J 1970;34:387-90.

12. Valentine VG, Raffin TA. The management of chylothorax. Chest 1992;102:586-91.

13. Yater WM. Non-traumatic chylothorax and chylopericardium; review and report of a case due to carcinomatous thromboangiitis obliterans of the thoracic duct and upper great veins. Ann Intern Med 1935;9:600-16.

14. Hasebrock K. Analysis of a chylose pericardial fluid (chylopericardium) [German]. Zeitschr Physiol Chemie 1888;12:289-94.

15. Han Z, Li S, Jing H, Liu H. Primary idiopathic chylopericardium: a retrospective case series. BMC Surg 2015;15:61. 